\title{
Smartphones in Surgery
}

\author{
Ara A. Salibian, BS; Thomas Scholz, MD* \\ Aesthetic \& Plastic Surgery Institute, University of California at Irvine, \\ California, USA
}

Submitted February 2011. Accepted for publication June 2011

\begin{abstract}
Smartphones have the capability of enhancing many aspects of the continuum of surgical care by providing an efficient means of multimedia communication among surgeons and healthcare personnel. The ability for mobile Internet and email access, along with features such as built-in cameras and video calling, allow surgeons to rapidly access, send and receive patient information without being restricted by issues of connectivity. Smartphones create an unrestricted network of data sharing, improving the flexibility of patient consultation, timeliness of preoperative preparation, efficiency of post-operative care and the effectiveness of a surgical team. Furthermore, smartphones provide mobile access to a multitude of surgical resources to bolster continued surgical education. This article presents a review of the current literature on the utilizations of smartphones in surgery, discusses their benefits and limitations, and addresses the possibility of incorporating smartphones into the protocol of surgical care.
\end{abstract}

Keywords: smartphone, pervasive computing, surgery, mobile health

\section{INTRODUCTION}

According to the International Programs Center, U.S. Census Bureau, the total population of the World, projected to September 2, 2011 is 6,959,479,878 [1]. There were 4.3 billion cell phone subscriptions in 2009, a number that is estimated to hit 5 billion at the end of 2010 [2,3]. Furthermore, the number of mobile broadband subscriptions will surpass 1 billion during the same year. These numbers reflect how much the world has changed with regards to the ways we communicate. Cell phones have been used globally to change our mobility, flexibility, availability, and our entire lifestyle in many different ways. Numerous organizations and commercial markets have understood the power of these changes impacting the end users on how to utilize these cell phones and smartphones.

*Corresponding Author: Thomas Scholz, University of California at Irvine, Aesthetic \& Plastic Surgery Institute, University of California, Irvine, $200 \mathrm{~S}$. Manchester Avenue, Suite 650, Orange, CA 92868.

Phone: (714) 456-5755. Fax: (714) 456-7718. Email: tscholz@uci.edu.

Other author: salibiaa@uci.edu 
The development of smartphones, or cell phones that provide advanced computing capabilities and Internet access, has helped to improve healthcare by allowing healthcare professionals to more efficiently communicate and share information. Though widely utilized in healthcare, smartphones are still a new and developing technology and the majority of the current literature is related to their general applications as communication and resource tools. Kailas et al. enumerate these applications, which include drug databases, medical calculators, reference programs, patient status tracking, communication, and patient record management [4]. Few articles deal with the particular applications of this mobile technology in surgical practice. Although surgeons do utilize many of the applications of smartphones also used by other physicians, tailoring smartphones to specifically meet the demands of surgeons is currently under investigation.

This article presents a review of the current literature on the uses of smartphones in surgery and communication, and focuses on how they can be adapted to improve the efficiency and versatility of surgical care. We will further analyze how cell phones and especially smartphones could change the field of surgery for health care professionals and patients alike.

The current review of literature was conducted by searching MEDLINE for articles published between 1990 and 2010 using the keywords "smartphone", "mobile healthcare", "telemedicine", and "teleconsultation". Literature selection was based on articles relevant to the utilization of smartphones anywhere in the surgical continuum of care. The engineering and technology database IEEE XPlore was also searched with the keywords "smartphone healthcare", "smartphone surgery", "telemedicine", and "teleconsultation" for articles from 1990 to 2010. Likewise, references found within relevant articles were also included.

\section{SMARTPHONES AND PERVASIVE COMPUTING}

Pervasive computing (PC) is an emerging field of mobile or embedded information and communication technologies with at least some degree of network connectivity and advanced user interface. Similar technologies that are loosely associated with and do not have an exact distinction from PC are ubiquitous computing and ambient intelligence [5-7]. In essence, $\mathrm{PC}$ represents the integration of computers into everyday aspects of life. The goal of PC is a seamless, natural and fluent interaction between technology and people to provide constant access to resources, whenever and wherever they are needed. Smartphones, like personal digital assistants (PDAs), laptops, and tablets, are examples of pervasive computing, that contribute to improving traditional healthcare via their ubiquitous, analytical, diagnostic, supportive, and documentary functions. Research in the field of pervasive computing for healthcare, however, is mainly focused on specific applications and proof of concepts rather than development of generic principles. Achieving these principles and analyzing critical success factors would require the knowledge of both engineers and health care professionals. 


\section{TELEMEDICINE}

\subsection{Telemedicine and Surgery}

Mirza et al. describes clinical and non-clinical mobile health applications with regards to disease management [8]. Clinical applications include access to databases, medication alerts, prescriptions via mobile phone, telemonitoring, transmission of test results, online health records, community nursing contact, public health and lifestyle messages, care of at-risk people, and emergency care. Non-clinical applications supported by mobile technologies are efficient wireless workflow, collection/sharing of data, asset utilization, patient or asset location, appointment booking, mobile phone support for patients and their caretakers, and safety of staff.

One of the most useful features of smartphones is their capability to optimize the practice of telemedicine, a developing practice that has been shown to improve patient care and hospital function [9]. Telemedicine is defined as the delivery of patient care using medical information that has been electronically communicated between two places [10]. In a specialty such as primary care, telemedicine is being used as a means of communication between physicians and patients [11]. In surgery, telemedicine can similarly be utilized for consultation, and can also be used to improve pre-operative and post-operative care. Inter-colleague communication and data sharing could help to improve the accuracy, efficiency, and thoroughness of surgical practice. The portability of smartphones and their ability to transmit data via satellite allows physicians to perform advanced computing and communication functions from any location, without having to locate a desktop computer or carry around a laptop. Smartphones therefore have the capability of greatly increasing the flexibility and utility of telemedicine, and through this ability to release audio, visual, and data information from wired and less portable platforms.

\subsection{Communication Forms and Content}

The possibility of rapid and unfettered data-sharing can prove useful in many scenarios in healthcare, particularly in an surgical environment where members of a surgical team, possibly including interns, residents, attending physicians and other staff, are optimally in constant communication with each other. Data shared with smartphones via email is only restricted by the parameters of regular email as smartphones have the same email capabilities as computers and can read most common image, video and document file formats such as jpeg, mp4, pdf and doc files. A surgeon can email patient chart data, X-rays, CT scans, high-resolution pictures and video as attachments in compliance with Health Insurance Portability and Accountability Act (HIPAA) protocol. Email accessibility can become an even more powerful tool when coupled with the ability to access electronic medical records (EMR) as well. This combination requires a wireless infrastructure throughout the hospital that would support such access from mobile devices. The possibility of accommodating such processes is increasing as hospitals are transitioning to EMR and are providing the means of networking to access these records remotely [12].

The ability to email almost any kind of patient data to and from a phone is invaluable in a setting where surgeons are always in transit between different geographic locations. Smartphones are also capable of improving the efficiency and speed of e-mail communication due to their increased portability. In this sense, smartphones may 
function as pagers that allow for rapid and extended communication among multiple surgeons. Furthermore, by using a proxy server that allows secure access to a hospital server from a remote location, this scenario can be extrapolated to any setting that has wireless availability or cellular reception.

The efficacy of all members of a surgical team within a hospital setting could be improved with the advent of smartphone technologies that allow locating and tracking of an individual at any time [13-15]. The entire team of physicians could be orchestrated by one oversight person who always keeps track of who is where and what everybody is doing at a given point in time. For example, if a doctor is needed in the outpatient clinic or the emergency department, that oversight person could redirect such a team member without delay. In emergency settings, this type of rapid response and improvement of communication can be a life-saving asset [16, 17]. PC is a technology that would play a center role in the feasibility of such a system.

In addition, the incorporation of advanced media features in smartphones, such as high-resolution cameras, abbreviates the process of sharing images taken with a standard camera. With smartphones, images can be taken, uploaded, emailed and viewed all with one portable device. Literature across different fields is fairly consistent in agreeing that the use of camera-phones for image capture and viewing is useful and efficient. Hsieh et al. reports using a camera phone for teleconsultation to successfully triage the majority of 80 cases of digital soft-tissue injuries [18]. Similarly, cameraphones have also been used to assess burns, blood smears, skin lesions and other visible maladies, with the general consensus from such studies that camera-phones prove valuable for image sharing due to factors of speed, efficiency, ease of use and portability [19-21]. The major evident drawback is the lower resolution of images taken by camera-phones along with viewing these images on their smaller screens [18-21]. However, a study conducted by Banitsas et al. on the use of mobile devices for teleconsultation concluded that the screen size and image quality on a PDA with a 3.8", $240 \times 320$ 16-bit color display were adequate for certain image viewing and videoconferencing functions [22]. Furthermore, current camera and display resolutions for smartphones are significantly greater than device specifications used in the aforementioned studies [23]. Different resolutions and screens sizes as well as other important specifications of smartphones for telemedicine are shown for popular smartphones in Table 1 [24-28].

Table 1. Comparison of relevant specifications of popular smartphones for telemedicine

\begin{tabular}{lccccc}
\hline Smartphone & $\begin{array}{c}\text { Screen Size } \\
\text { (inches) }\end{array}$ & $\begin{array}{c}\text { Display } \\
\text { (pixels) }\end{array}$ & $\begin{array}{c}\text { Camera } \\
\text { (megapixels) }\end{array}$ & $\begin{array}{c}\text { Video Calling } \\
\text { (Yes/No) }\end{array}$ & $\begin{array}{c}\text { Video Capture } \\
\text { (pixels) }\end{array}$ \\
\hline iPhone 4® & 3.5 & $960 \times 640$ & 5 & Yes & $720 \mathrm{p} \mathrm{HD}$ \\
EVO 4G & 4.3 & $480 \times 800$ & 8 & Yes & $720 \mathrm{p} \mathrm{HD}$ \\
DROID Incredible ${ }^{\circledR}$ & 3.7 & $480 \times 800$ & 8 & No & $720 \mathrm{p} \mathrm{HD}$ \\
BB ${ }^{\circledR}$ Torch 9800 & 3.2 & $369 \times 480$ & 5 & No & $640 \times 480$ \\
Nokia ${ }^{\circledR}$ n8 & 3.5 & $360 \times 640$ & 12 & Yes & $720 \mathrm{p} \mathrm{HD}$ \\
\hline
\end{tabular}

iPhone is a trademark of Apple Inc. EVO 4G is a trademark of HTC Corporation. DROID is a trademark of Lucasfilm Ltd. and its related companies. Incredible by HTC is a trademark of HTC Corporation. BB (BlackBerry) is a trademark of RIM. Nokia is a trademark of Nokia Corporation. 
The cameras recommended for telemedicine, however, all have resolutions around 12 megapixels [29]. Therefore, the decision to use the option of capturing images in addition to sending and receiving them on a smartphone must be dictated by the situation that requires such image sharing. Any surgical specialty dealing with visible pathology or trauma can benefit from this technology. Evidently, the photo-taking capabilities of smartphones would be most useful when a scenario necessitates rapid feedback. For example, a surgery resident in the emergency room could take a picture of a wound and immediately send it to the attending physician for advice. Similarly, intra-operative images could be taken and quickly emailed to many residents, attendings or other surgeons for discussion [30]. The need for rapid assessment of images by a physician remotely, or not physically present, provides an opportunity for the use of the integrated camera on the smartphone to surpass standard photography and uploading procedures. Furthermore, the addition of imaging information in combination with patient data, EMR, rapid exchange with colleagues potentiates the value for the responsible physician and provides a new platform of rapid and effective response.

The introduction of video-calling features further increases the potential of smartphones to function as a thorough, all-in-one method of communication among surgeons. Instead of having to assess a situation with information given solely over the phone, or even looking at emailed images and then discussing a case over the phone, an attending surgeon can answer a question about a visible problem while simultaneously viewing the site in question from any angle that the smartphone holder chooses. The spectrum of scenarios in which this technology could be employed appears very broad. Smartphone video-calling could also be used in a number of other situations such as general symptom evaluation. This video-calling function in general has already been implemented successfully in hospital interpreter services. Both videoconferencing and hands-free telephones can deliver an acceptable interpreting service compared to a physically present interpreter with the advantage of time efficiency and cost effectiveness [31]. Similar availability of surgeons via video-calling for patients, colleagues, and team members could hold some promise with regards to efficiency, time management, safety, and cost effectiveness. However, the authors would like to emphasize that this method of communication should not, of course, replace any situation that warrants the physical presence of an attending or any other surgical authority. It should instead be used to supplement appropriate conversations. Finally, the camera or video function of smartphones could be a helpful asset in triage of patients. Patients calling their doctors or even emergency numbers could be advised and directed in a more comprehensive manner.

\subsection{Consultation}

Accessing information from a smartphone allows the physician to view emails, images, videos, patient data, or other information that can be retrieved from the Internet, anytime during the day and at any location. The ease of email access along with the portability of the smartphone makes it a useful tool for quickly relaying data that can be used to confer with colleagues on consultations. This may prove especially useful in an academic environment where surgical interns and residents can email questions on 
consultations supplemented with visual data, improving the ability of attendings to efficiently respond from a remote location. Analyzing a wound or surgical incision and finding an appropriate treatment option is individual and often times lacks treatment consensus. The immediate exchange with other surgeons is a helpful adjunct to their armada of treatment options. This rapid sharing of data greatly enhances the communication between surgeons and may improve the efficiency of choosing proper surgical treatment for patients.

Aziz et al. demonstrate the simplicity and efficiency of such communication in sharing digital imaging information for oral and maxillofacial surgery cases [32]. In this study, images are downloaded onto a desktop computer from a Picture Archiving and Communication System (PACS) server, converted into viewable formats, and emailed to faculty who can access the data from their smartphones. The authors present a case in which an incorrect diagnosis of a nondisplaced orbital floor fracture was made, and the correct diagnosis of a left zygomatic/maxillary complex fracture was made by an away attending after the emailed CT scan was viewed on his smartphone. Accessing these images remotely decreased the time necessary to provide the correct diagnoses and therefore proper treatment.

\subsection{Pre-Operative Management}

In addition to offering the rapid exchange of multimedia information, smartphones also provide the opportunity for the mass sharing of information, a feature that would be particularly useful for all members of a surgical healthcare team in the operating room (OR). Hospital ORs represent a center of efficiency, coordination, safety, and precision, and a time-efficient and direct communication style is critical. A recent study showed that around fifty percent of operations have delays, and though intra-operative times contribute to this number, pre- and post-operative changing times take up a significant amount of overall OR time [33-35]. It is during these times that the first changes should be made to improve efficiency in the OR. The average OR starts on time only 27 percent of the time [35]. There are many factors that contribute to this delay such as mechanical malfunction and anesthesia problems; however, one of the easiest causes that can be fixed is poor communication. A previous study showed that smartphones given to members of health care and nursing teams increase team communication and support faster delivery of healthcare [36]. The same phenomenon can be translated to benefit a surgical team in the OR to allow for more efficient management of time in between cases. By allowing rapid two-way exchange of information among surgeons, anesthesiologists, and nurses, the smartphone could potentially solve some of the communication problems that contribute to late start times. From a surgeon's perspective, pre-operative improvement could include tracking of patients via smartphone. The surgeon would be able to see if the patient is still in the pre-operative holding area, on the way to the OR, or already in the OR. Simple transmission of a video-call to his phone would give him necessary information about the status in the room and so would be able to enter the OR in a timely manner without wasting important time. Furthermore, with two-way communication through such an interface, the attending surgeon could manage the workings of the OR before arriving there, such as requesting certain instruments and making sure certain people are there. In addition, 
messaging to patients could result in a reduced number of missed outpatient surgery procedures as already seen for non-surgical hospital appointments [8].

An EMR access feature, as previously mentioned, could also be incorporated into the program, putting all the information the surgeon needs in one place, and more importantly, a place that can be accessed wherever the surgeon may be before the case. This combination of communication provides an interface that connects all personnel in the OR and could possibly eliminate much of the unnecessary time spent remediating the effects of under preparedness and miscommunication. Ideally, a study modeled after the one of [36] would be able to determine whether mobile accessibility to patient data and the OR could improve pre-operative efficiency and safety.

\subsection{Post-Operative Care}

Post-operative care is an essential component of the surgical continuum that requires diligent monitoring of patient's labs, wounds and general condition. The attending surgeon's post-operative contact with the patient unfortunately can be limited at times. Therefore, the surgeon's understanding of the patient's status is dependent on nurses and doctors communicating effectively with each other. Smartphones could help with the surgeon's availability and communication among team members including data transmission. In an academic setting, the presence of multiple residents and interns increases the size of the team making effective communication even more challenging. Certain factors involved in patient monitoring, such as lab values and the flow chart, do not pose problems when restricted to communication over the phone. However, there are also many physical descriptors of patient's status, such as condition of the operative site that require subjective interpretation by the surgeon. Frequently, communication over the phone does not replace objective visualization by the surgeon, and results in the surgeon having to come in to see the patient in person because of the difficulty of communicating these descriptors verbally.

The smartphone can provide a simple solution to this problem as it combines the mediums of verbal and media communication in a mobile interface. Binder et al. present a study on the use of teledermatology for wound management of 16 patients with leg ulcers by home care nurses, concluding that this method of treatment could clearly increase the quality of medical care [37]. The video-calling feature of smartphones functions is a similarly useful tool as it allows healthcare personnel at the hospital to easily maneuver the camera of the smartphone around an area in question while simultaneously communicating with the surgeon who can request almost any viewing angle from the portable device. Furthermore, this feature is able to capture and transmit valuable dynamic examinations, such as capillary refill of tissue or blanching versus non-blanching erythema.

Though wireless connectivity in hospitals is becoming more common, finding a wireless connection outside of a hospital, residence, or business can be difficult. The portability of the smartphone takes this concept of telemonitoring a step further by freeing the surgeon from the constraints of having to find a hardwired machine or wireless connection to complete such a procedure while away from the hospital. This prevents unnecessary trips to the hospital and improves the standard of patient care while avoiding the confusion and misunderstanding in communication restricted by the 
telephone. This problem has been partially solved in the past with remote access via Internet into hospital systems to allow physicians to view data, imaging studies, and patient information from home. The authors feel that taking the next step and providing this type of remote access via the physician's smartphone not only improves effectiveness, but also increases patient safety by giving the surgeon a more complete and accurate understanding of the patient's condition as problems can be visualized directly, and the need to relay information through a third party is eliminated. However, it is important that video-calling is not used to pass final judgment on a case in question even after mobile viewing, and that a physical visit always occurs in a situation of uncertainty. Surgeons could also utilize programs that continually update patient vitals and other stats from machines instead of having to call and find a nurse to obtain such information. For example, the surgeon could log in the program and check the patient's flowchart. This would not only save time for the physician, but also free up nurse's time to take care of other tasks. The smartphone would give the surgeon complete and definitive surveillance of a patient's postoperative condition through a mobile platform that enables the surgeon to be immediately updated on a patient's status. Residents are frequently confronted with clinical situations where guidance by an attending physician is required. Taking a picture with the smartphone of a patient's EKG or surgical wound in the postoperative period during house night calls would be helpful, but is not an accepted method and does not comply with patient's confidentiality by any means. In short, the technology exists, but that is the easiest part.

Taking the smartphone technology, adjusting it to surgical needs and making it feasible, secure, and safe remains a future challenge. For instance, after performing a buried free flap with an implantable Doppler [38], the surgeon can call the intensive care unit on the evening after the surgery and have the nurse hold the phone to the Doppler probe so that the Doppler signal can be heard through the phone [39] . This represents very important first-hand information for the surgeon in an early postoperative phase. Smartphones may provide the opportunity for the surgeon to receive additional information from this procedure, such as the ability to visualize Doppler waveforms or color Dopplers. Improving this kind of technology, rather than holding a phone next to a medical Doppler device, can improve the quality and thus the safety of post-operative management. Again, this kind of innovation most probably would be a conjoint effort that brings together the needs and ideas of a clinician and the technical expertise of an engineer.

\section{SURGICAL EDUCATION}

Besides its ability to enhance communication, the smartphone also serves as an invaluable educational resource, not only in surgical training, but in continuing medical and surgical practice. In the simplest manner, the smartphone provides mobile access to the Internet, allowing the surgeon to access a countless number of resources whenever and wherever there is a wireless connection and cellular reception. This creates a condensed, on-the-go reference library that can be used to research any topic or question immediately and rapidly. The extensive media-viewing capabilities of smartphones allow a surgeon to view a variety of different learning resources, whether it is an article or an instructional video. 
In addition, many smartphones have incorporated the ability to download add-on applications [40]; however, the current discussion will focus on the applications available for the iPhone, as it currently provides the widest variety of add-on's and the iTunes Store is actually the largest media seller in the world [41]. These applications can be made by third-party developers, who have created a wide variety of medical applications, many of which are useful for surgeons. Simbionix, for example, a company that designs virtual reality simulators to train medical professionals, started a Medical Education Division through eTrinsic, a division of Simbionix ${ }^{{ }^{\circledR *}}$ for online education [42]. The company is currently developing mobile applications for physicians that, among many other collaboration and educational materials, provide in-depth virtual procedure training programs. Some of the already-released applications include a course on inguinal hernia repair, umbilical hernia repair, and surgical wound healing. The ability to access such didactic information from the iPhone extends learning beyond the classroom and the wards, enabling training surgeons to constantly review and stay up-to-date with important educational material.

Many other surgical applications are also available for educational and reference purposes, as well as for convenience. I-surgery Notebook $^{\circledR \dagger}$, for example, is an application that allows the surgeon to keep a log of surgical cases by inputting patient and procedure data, including images, for each case into the program [43]. An advanced search feature then allows the surgeon to quickly search and review case logs. Surgery On Call ${ }^{\odot \ddagger}$ is a reference application that has a thorough catalog of information on the clinical evaluation and management of surgical cases [44]. Other programs, such as anatomy applications are also available, and provide an easy and efficient way for a surgeon to reference anatomy atlases for procedures. In addition, there are a number of podcasts that can be downloaded straight onto the iPhone for listening anywhere. These consist of everything from an audio book on complications in surgery to a monthly podcast review of cornea and refractive surgery articles $[45,46]$. The possibilities for different mobile surgical applications are numerous, and with the help of a rapidly growing database of educational, reference, and interactive applications, surgeons will be able to provide smoother, easier, and more efficient healthcare. On the other side, patient education via smartphones is an emerging concept that is of high importance in the entire medical field. Mobile health technologies have been proven to support the transition to the patient empowerment model [47]. It requires patients to be knowledgeable so that they can make choices on how to manage their health and creates a shift from a traditional biomedical model to a more integrated concept with clinicians and patients as rather equal partners during the decision making process [48].

\footnotetext{
*1Simbionix is a trademark of the Simbionix USA Corporation. $\dagger$ I-Surgery Notebook is a copyright of IsurgeryNotebook.com. $¥$ Surgery On Call is a copyright of Current Clinical Strategies Publishing.
} 


\section{CRITICAL ISSUES}

There still are limitations, however, to the use of smartphones in clinical and hospital settings. The major concern with regard to accessing, storing or transmitting clinical information is the protection of personal health information. Security threats related to the mobile access of personal health information must be thoroughly addressed prior to the allowance of mobile access to patient records. Bones et al. presented a risk analysis for the use of smartphones in healthcare, stating that the web-access and email features of the phones should be disabled while the devices are used in a healthcare setting as these features are the primary targets for security risks [49]. However, the protection of electronically transmitted personal health information via such avenues has been regulated by HIPAA Security Act guidelines, a protocol that was created in 2003 to protect the transmission and access of "electronic protected health information" (e-PHI) [50].

The protection of such information parallels the protection of any e-PHI accessed from the smartphone, whether through email or applications, in that it must be compliant with HIPAA Security Act guidelines for access management, backup, encryption and other enforcements. The ability to access this information from smartphones poses an even greater risk as phones can more easily get into unauthorized hands than a computer. Criteria that ensure that e-PHI on a smartphone is secure and HIPAA-compliant include password protection of the device, limiting the email stored on the device to no more than 250 messages or 7 days, support for data encryption, and the ability for data on the smartphone to be remotely erased [51]. Likewise, access to any applications on the phone that are connected to EMR should have further encryption. Furthermore, patient consent should be obtained before any electronic protected health information is downloaded to a smartphone. Even with adherence to such regulations, security risks associated with hacking and data infiltration are always present when Internet access is involved.

Other issues with smartphone usage include the practicality of Internet access from smartphones, which requires a wireless connection (preferably) or cellular reception to transmit and receive data. With many hospitals providing total wireless coverage through their institutions, access within hospitals should be feasible in the near future [52]. Outside of the hospital, however, coverage varies depending on service providers, an issue that can be taken into account when choosing the proper smartphone. Furthermore, it is imperative to understand when it is appropriate to use smartphones for these purposes, making sure that certain scenarios, such as using a smartphone while with a patient or while driving, are avoided.

It is important to recognize the limitations of smartphones in determining the efficiency and practicality of different computing devices for telemedicine functions in specific scenarios. Desktop computers, for example, may be a preferred option when available as they have large screens, more computing functions than portable devices, and may allow for work to be completed faster than on smaller devices. Portable devices, on the other hand, should be considered when computer terminals are not conveniently and locally available. Tablet computers, for example, are portable devices that offer equivalent if not greater computing capabilities than smartphones and are recently being equipped with many of the same features that make smartphones useful for telemedicine, such as front-facing cameras for video-calling. In certain situations, 
such as when images need to be analyzed in detail, or longer tasks need to be performed on these devices, smartphones may fall short of tablets as their small screen and keyboard size can make certain tasks more difficult and impractical. However, smartphones may be a better alternative in other scenarios, such as when rapid assessment is needed. In addition, a key feature of smartphones is their portability and expanded connectivity through the use of data plans. Though tablets are smaller than laptops, they still cannot be carried around with the same ease as smartphones, and would require an additional data subscription to prevent reliance on wireless connections for communication. These different devices do not necessarily have to be isolated and contrasted with each other, as they may serve complementary functions depending on the different situations in which they function best. Further research will be required, after integration of newer devices such as smartphones and tablets into healthcare protocol, to determine how to choose among these options in the appropriate scenarios.

These issues, and the many more that can arise when using smartphones in a medical setting, point to the need for the creation of a standard protocol for using smartphones in the clinical setting. Furthermore, introducing smartphones and mobile technologies into the working day of surgeons with limitless availability could decrease uninterrupted time with patients. It is important to understand that when utilizing these new technologies in our everyday lives, they should not replace or limit the value of a personal, empathetic face-to-face conversation.

\section{CONCLUSION}

Smartphones are an integral part of the social mainstream and are integrated into many aspects of society. Smartphones can provide a superior method of communication and function as an important clinical tool for surgeons by offering mobile and efficient data sharing among colleagues and healthcare staff, as well as portable access to a wealth of critical information. The data capturing and sharing features of smartphones are especially beneficial for surgeons who deal with externally visible conditions. Smartphones may provide even more individual applicability and functionality for the surgeon than EMR systems. Continuous monitoring of patients, interactive consultation, rural and remote care, as well as fast emergency responses via smartphones will soon be available. Further research is needed to evaluate the applicability of these newly developed mobile health technologies for surgeons, health care workers, and patients to reshape and adjust the field of surgery.

\section{CONFLICT OF INTEREST: None.}

\section{REFERENCES}

[1] U.S. Census Bureau. International Data Base. 2011; http://www.census.gov/ipc/www/idb/worldpopinfo.php. Accessed May 5, 2011.

[2] International Telecommunications Union. ICT Statistics - Mobile cellular subscriptions. 2009.http://www.itu.int/ITUD/icteye/Reporting/ShowReportFrame.aspx?ReportName=/WTI/Cellular SubscribersPublic\&ReportFormat=HTML4.0\&RP_int Year=2009\&RP_intLanguageID=1\&RP_bitLi veData=False. Accessed November 1, 2010. 
[3] International Telcommunications Union. ITU sees 5 billion mobile subscriptions globally in 2010. 2010. http://www.itu.int/newsroom/press_releases/2010/06.html. Accessed November 1, 2010.

[4] Kailas A, Chong CC, Watanabe F. From mobile phones to personal wellness dashboards. IEEE Pulse, 2010, 1(1): 57-63.

[5] Hansen TR, Bardram JE, Soegaard M. Moving out of the lab: Deploying pervasive technologies in a hospital. IEEE Pervasive Computing, 2006, 5(3): 24-31.

[6] Weiser M. The Computer for the 21st-Century. Scientific American, 1991, 265(3): 94-104.

[7] IST Advisory Group. Scenarios for ambient intelligence in 2010. Final Report. Seville: European Commission Joint Research Centre, Institue for Prospective Technological Studies (IPTS). 2001.

[8] Mirza F, Norris T, Stockdale R. Mobile technologies and the holistic management of chronic diseases. Health Informatics Journal, 2008, 14(4): 309-21.

[9] Lilly CM, Cody S, Zhao H, Landry K, Baker SP, McIlwaine J, Chandler MW, Irwin RS. Hospital mortality, length of stay, and preventable complications among critically ill patients before and after tele-ICU reengineering of critical care processes. Journal of the American Medical Association, 2011, 305(21): 2175-2183.

[10] American Telemedicine Association. Telemedicine Defined. 2011.http://www.americantelemed.org/i4a/pages/index.cfm?pageid=3333. Accessed May 10, 2011.

[11] McKinstry B, Watson P, Pinnock H, Heaney D, Sheikh A. Telephone consulting in primary care: a triangulated qualitative study of patients and providers. British Journal of General Practice, 2009, 59(563): e209-18.

[12] Gamble KH. Beyond phones. With the proper infrastructure, smartphones can help improve clinician satisfaction and increase EMR use. Healthcare Informatics, 2009, 26(8):23-4, 26.

[13] Schrooyen F, Baert I, Truijen S, Pieters L, Denis T, Williame K, Weyn M. Real Time Location System over WiFi in a Healthcare Environment. The Journal on Information Technology in Healthcare, 2006, 4(6): 401-416.

[14] Bardram JE. Activity-based support for mobility and collaboration in ubiquitous computing. Ubiquitous Mobile Information and Collaboration Systems, 2004, 3271:166-80.

[15] Drugge M, Hallberg J, Parnes P, Synnes K. Wearable systems in nursing home care: Prototyping experience. IEEE Pervasive Computing, 2006, 5(1): 86-91.

[16] Chen CI, Choy CS, Chu TB, Lo YS, Huang WK, Kuan CF, Li YC. Safety portal: the safest goes through the air-ubiquitous high-risk reminders bridging out the patient safety in emergency department. Stud Health Technol Inform, 2006, 124:77-82.

[17] Chen CI, Liu CY, Li YC, Chao CC, Liu CT, Chen CF, Kuan CF. Pervasive Observation Medicine: The Application of RFID to Improve Patient Safety in Observation Unit of Hospital Emergency Department. Studies in Health Technology and Informatics, 2005, 116: P311-15.

[18] Hsieh CH, Tsai HH, Yin JW, Chen CY, Yang JC, Jeng SF. Teleconsultation with the mobile cameraphone in digital soft-tissue injury: a feasibility study. Plastic and Reconstructive Surgery, 2004, 114(7): 1776-82.

[19] Chung P, Yu T, Scheinfeld N. Using cellphones for teledermatology, a preliminary study. Dermatology Online Journal, 2007, 13(3):2.

[20] McLean R, Jury C, Bazeos A, Lewis SM. Application of camera phones in telehaematology. Journal of Telemedicine and Telecare, 2009, 15(7): 339-43.

[21] Shokrollahi K, Sayed M, Dickson W, Potokar T. Mobile phones for the assessment of burns: we have the technology. Emergency Medicine Journal, 2007, 24(11):753-5.

[22] Banitsas KA, Georgiadis P, Tachakra S, Cavouras D. Using handheld devices for real-time wireless teleconsultation. Conference Proceedings: Annueal International Conference of the IEEE Engineering in Medicine and Biology Society, 2004, 4: 3105-8.

[23] Edwards C. The killer iPhone. How Apple designed its new model to be sweet to customers an vicious to rivals. Strategic Direction, 2010, 26(11): 68-69. 
[24] Apple Inc. iPhone 4 Technical Specifications. 2011. http://www.apple.com/iphone/specs.html. Accessed May 10, 2011.

[25] HTC. EVO 4G Tech Specs. 2011. http://www.htc.com/us/products/evo-sprint/\#tech-specs. Acessed May 10, 2011.

[26] HTC. DROID Incredible by HTC Tech Specs. 2011. http://www.htc.com/us/products/droid-incredibleverizon\#tech-specs. Accessed May 10, 2011.

[27] Research in Motion. Blackberry Torch 9800 Features. 2011. http://us.blackberry.com/smartphones/blackberrytorch/\#!phone-specifications. Acessed May 10, 2011.

[28] Nokia Corporation. Nokia n8 Specifications. 2011. http://www.nokiausa.com/findproducts/phones/nokia-n8-alt/specifications. Accessed May 10, 2011.

[29] Patricoski C, Ferguson AS, Brudzinski J, Spargo G. Selecting the right digital camera for telemedicinechoice for 2009. Telemedicine Journal and E-health, 2010, 16(2): 201-8.

[30] Lichtor JL. Camera phone as the medium. Anesthesiology, 2010, 112(1): 196.

[31] Jones D, Gill P, Harrison R, Meakin R, Wallace P. An exploratory study of language interpretation services provided by videoconferencing. Journal of Telemedicine and Telecare, 2003, 9(1): 51-6.

[32] Aziz SR, Ziccardi VB. Telemedicine using smartphones for oral and maxillofacial surgery consultation, communication, and treatment planning. Journal of Oral and Maxillofacial Surgery, 2009, 67(11):2505-9.

[33] Harders M, Malangoni MA, Weight S, Sidhu T. Improving operating room efficiency through process redesign. Surgery, 2006, 140(4):5 09-14; discussion 514-6.

[34] Healthcare Financial Management A. Achieving operating room efficiency through process integration. Healthcare Financial Management, 2003, 57(3): suppl 1-7 following 112.

[35] Wong J, Khu KJ, Kaderali Z, Bernstein M. Delays in the operating room: signs of an imperfect system. Canadian Journal of Surgery, 2010, 53(3): 189-95.

[36] O'Connor C, Friedrich JO, Scales DC, Adhikari NK. The use of wireless e-mail to improve healthcare team communication. Journal of the American Medical Informatics Association, 2009, 16(5): 705-13.

[37] Binder B, Hofmann-Wellenhof R, Salmhofer W, Okcu A, Kerl H, Soyer HP. Teledermatological monitoring of leg ulcers in cooperation with home care nurses. Archives of Dermatology, 2007, 143(12): 1511-4.

[38] Paydar KZ, Hansen SL, Chang DS, Hoffman WY, Leon P. Implantable venous Doppler monitoring in head and neck free flap reconstruction increases the salvage rate. Plastic and Reconstructive Surgery, 2010, 125(4): 1129-34.

[39] K. Paydar, personal communication, October 2010.

[40] Burdette SD, Herchline TE, Oehler R. Surfing the web: practicing medicine in a technological age: using smartphones in clinical practice. Clinical Infectious Disease, 2008, 47(1): 117-22.

[41] Oehler RL, Smith K, Toney JF. Infectious diseases resources for the iPhone. Clinical Infectious Disease, 2010, 50(9): 1268-74.

[42] Symbionix. 2010. www.etrinsic.com. Accessed October 25, 2010.

[43] I-Surgery Notebook. 2010. http://www.lifewareapps.com/isn/. Accessed October 25, 2010.

[44] Surgery on Call. 2010. http://itunes.apple.com/us/app/surgery-on-call/id387751948?mt=8. Accessed October 25, 2010.

[45] Gawande, A. Complications: a surgeon's notes on an imperfect science. 2003. www.audible.com. Accessed November 1, 2010.

[46] Morshifar M, Khalifa Y. Cornea and refractive surgery update. 2009. http://itunes.apple.com/us/podcast/cornea-refractive-surgery/id330828691. Accessed November 1, 2010.

[47] Connelly KH, Faber AM, Rogers Y, Siek KA, T T. Mobile applications that empower people to monitor their personal health. Elektrotechnik und Informationstechnik, 2006, 123(4):124-128.

[48] Winkelman WJ, Choo CW. Provider-sponsored virtual communities for chronic patients: improving health outcomes through organizational patient-centred knowledge management. Health Expectations, 2003, 6(4): 352-8. 
[49] Bones E, Hasvold P, Henriksen E, Strandenaes T. Risk analysis of information security in a mobile instant messaging and presence system for healthcare. International Journal of Medical Informatics, 2007, 76(9): 677-87.

[50] Centers for M, Medicaid Services HHS. Health insurance reform: security standards. Final rule. Federal Registrar, 2003, 68(34):8334-81.

[51] Yale University. Health insurance portability and accountability act. 2010. http://www.yale.edu/hipaa/solutions/smartphones.html. October 25, 2010.

[52] Gamble KH. Wireless Tech Trends 2010. Trend: smartphones. Healthcare Informatics, 2010, 27(2): 24, 26-7. 


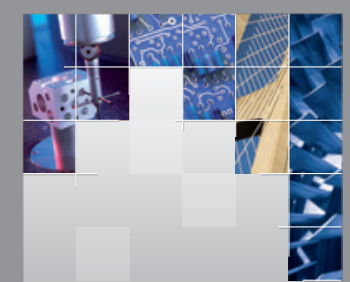

\section{Enfincering}
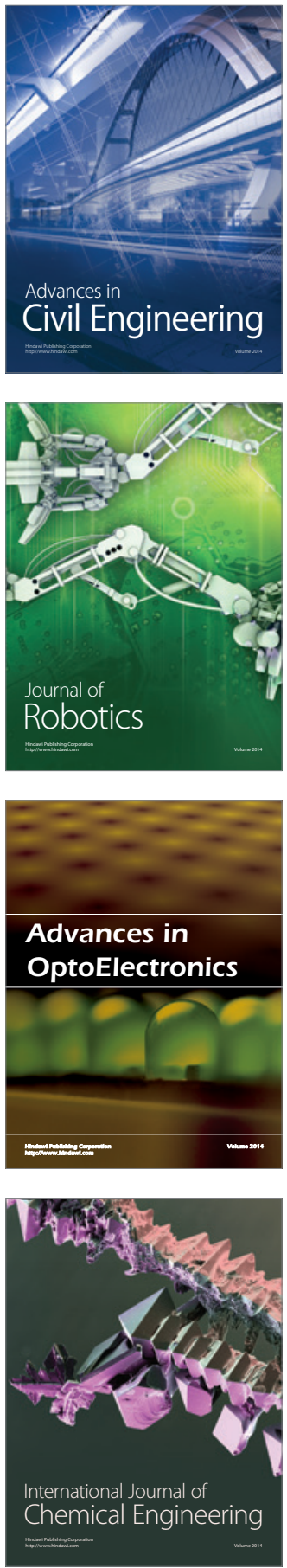

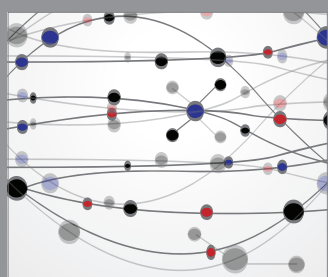

The Scientific World Journal

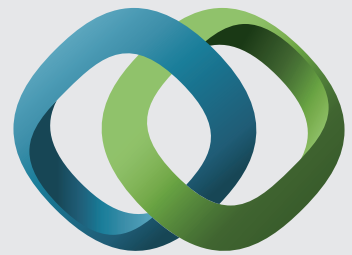

\section{Hindawi}

Submit your manuscripts at

http://www.hindawi.com
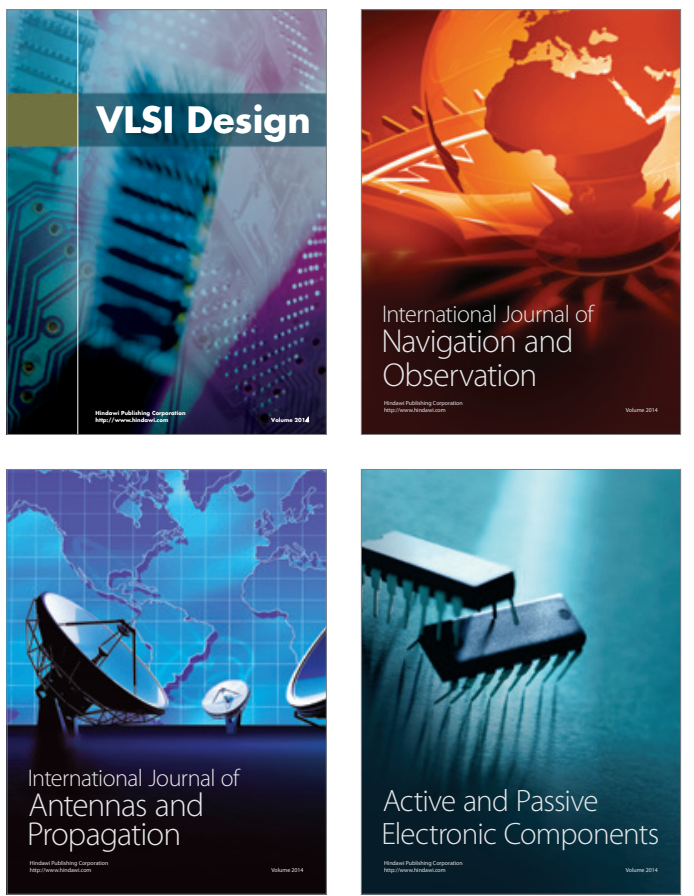
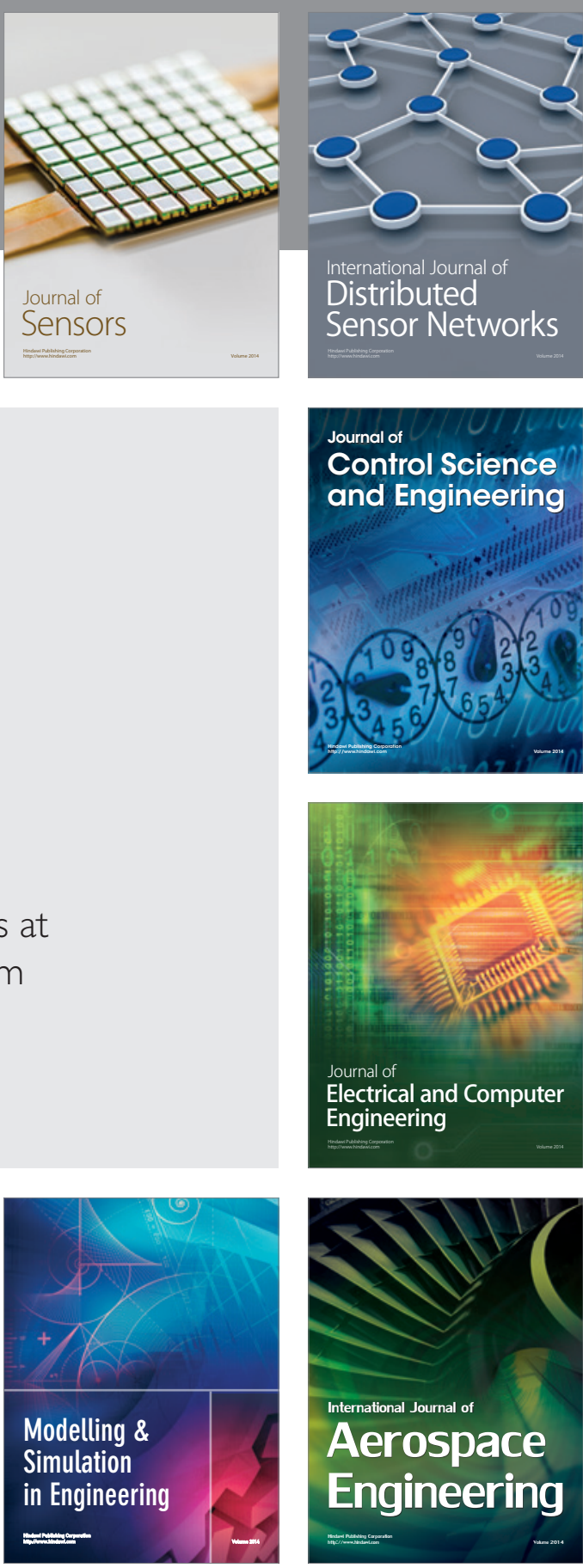

International Journal of

Distributed

Sensor Networks

Journal of

Control Science

and Engineering
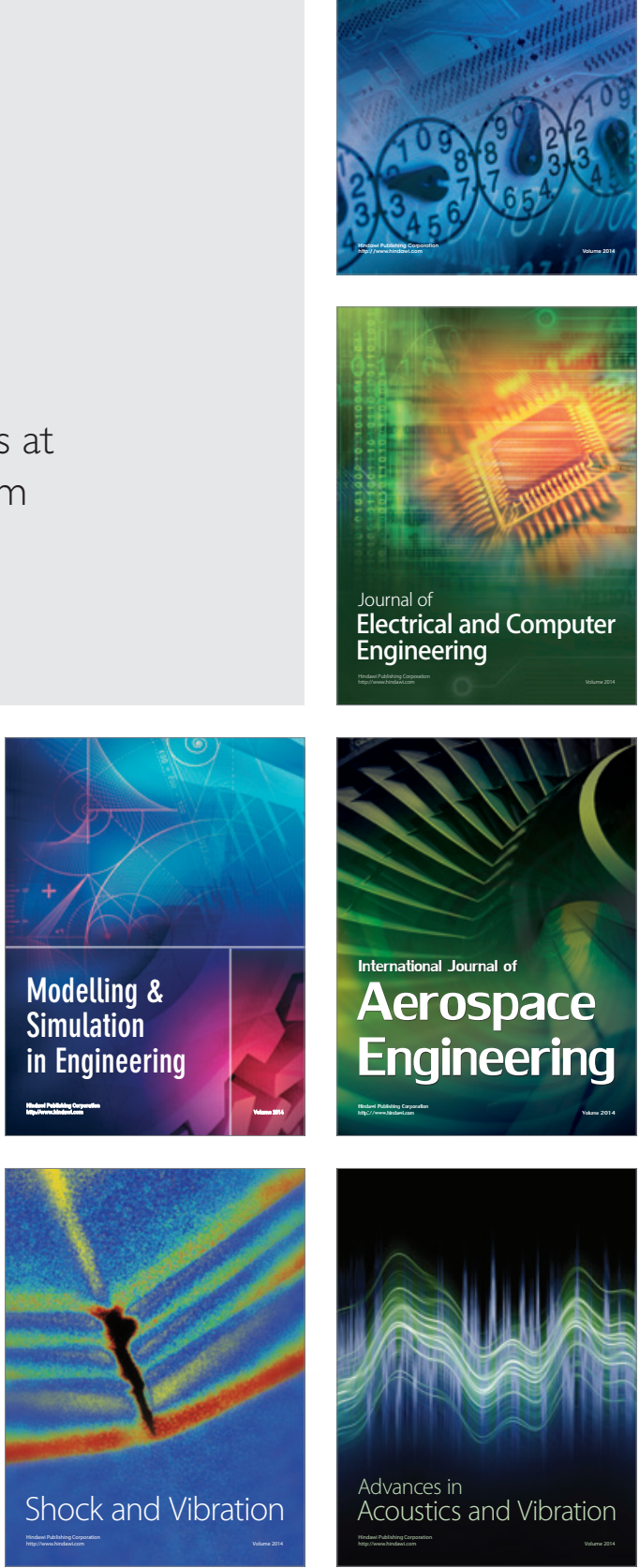